\title{
On the Inversion of Certain Matrices
}

\author{
By Samuel Schechter
}

1. Introduction. Let $a_{1}, a_{2}, \cdots, a_{n} ; b_{1}, b_{2}, \cdots, b_{n}$ be $2 n$ distinct, but otherwise arbitrary, complex numbers. For the matrix $H$, of order $n$,

$$
H=\left\{\frac{1}{a_{i}-b_{j}}\right\} \quad \text { with } 1 \leqq i, j \leqq n
$$

let $G=H^{-1}=\left\{c_{i j}\right\}$. (The indices $i, j, k$ will range from 1 to $n$ unless it is specified otherwise.) If, for some constant $p$,

$$
a_{i}-b_{j}=i+j-1+p \neq 0,
$$

then $H$ is a segment of the well known generalized Hilbert matrix, and in this case formulas for $c_{i j}$ have been given by Savage and Lukacs [4], Smith [6] and Collar [1]. For

$$
a_{i}-b_{j}=i-j+p
$$

Linfoot and Shepherd [3] and Collar [2] give formulas for $c_{i j}$ and in both cases Collar exhibits diagonal matrices $D, K$ such that $G=D H^{r} K$. Collar [2] and Smith also evaluate the quantities $\sum_{i, j} c_{i j}, \sum_{j} c_{i j}$.

These authors make use, in most cases, of the formula for the determinant [5]

$$
\operatorname{det} H=\frac{\prod_{j>k}\left(a_{j}-a_{k}\right)\left(b_{k}-b_{j}\right)}{\prod_{j, k}\left(a_{j}-b_{k}\right)}
$$

or require the evaluation of certain involved series.

The formulas of Collar and Smith are extended here to the general case (1). The method to be used does not depend on (4) but simply on Lagrange's interpolation formula. Indeed (4) comes out as a by-product of formula (17) given below.

\section{Formula for the inverse. Let}

$$
A(x)=\prod_{i}\left(x-a_{i}\right), \quad B(x)=\prod_{i}\left(x-b_{i}\right)
$$

and denote the fundamental polynomials of the Lagrangian interpolation corresponding to the $a_{i}$ and $b_{i}$, respectively, by

$$
A_{i}(x)=\frac{A(x)}{A^{\prime}\left(a_{i}\right)\left(x-a_{i}\right)}, \quad B_{i}(x)=\frac{B(x)}{B^{\prime}\left(b_{i}\right)\left(x-b_{i}\right)}
$$

where prime denotes differentiation. We then have

Received February 13, 1959. The work for this paper was done at the AEC Computing and Applied Mathematics Center, Institute of Mathematical Sciences, under a contract with the U. S. Atomic Energy Commission. 
Theorem 1. The elements of $G$ are given by

$$
c_{i j}=\left(a_{j}-b_{i}\right) A_{j}\left(b_{i}\right) B_{i}\left(a_{j}\right)
$$

and if $H$ is symmetric

$$
c_{i j}=\left(a_{j}-b_{i}\right) A_{j}\left(b_{i}\right) A_{i}\left(b_{j}\right) .
$$

Proof: For any polynomial $p(x)$ of degree $n-1$ we may write

$$
p(x)=\sum_{i} p\left(a_{i}\right) A_{i}(x)
$$

or

$$
\frac{p(x)}{A(x)}=\sum_{i} \frac{c_{i}}{x-a_{i}}
$$

where

$$
c_{i}=\frac{p\left(a_{i}\right)}{A^{\prime}\left(a_{i}\right)} .
$$

Now let $p(x)=-B_{k}(x) A\left(b_{k}\right)=p_{k}(x)$ and

Then from (10) we obtain

$$
c_{k i}=\frac{p_{k}\left(a_{i}\right)}{A^{\prime}\left(a_{i}\right)} .
$$

$$
\frac{B_{k}(x) A\left(b_{k}\right)}{A(x)}=\sum_{i} \frac{c_{k i}}{a_{i}-x} .
$$

If we set $x=b_{j}$ then, since $B_{k}\left(b_{j}\right)=\delta_{k j}$, the Kronecker delta, we get that

$$
\delta_{k j}=\sum_{i} \frac{c_{k i}}{a_{i}-b_{j}} .
$$

Thus $c_{k i}$ gives the desired inverse element, that is,

$$
c_{k i}=\frac{-B_{k}\left(a_{i}\right) A\left(b_{k}\right)}{A^{\prime}\left(a_{i}\right)}=\left(a_{i}-b_{k}\right) B_{k}\left(a_{i}\right) A_{i}\left(b_{k}\right) .
$$

If $H$ is symmetric: $a_{i}-b_{j}=a_{j}-b_{i}$, it follows that $B_{i}\left(a_{j}\right)=A_{i}\left(b_{j}\right)$ and the theorem is proved.

We now obtain a simple formula for the sum of the $c_{i j}$. This quantity arises in problems of aerodynamics [2] and its simplicity allows it also to be used as a check on some alleged inverse.

Corollary.

$$
\sum_{i, j} c_{i j}=\sum_{k}\left(a_{k}-b_{k}\right)=s
$$

Proof. If we apply (9) to $p(x)=B_{j}(x)$ and set $x=b_{k}$ we obtain that

$$
\sum_{i} B_{j}\left(a_{i}\right) A_{i}\left(b_{k}\right)=\delta_{j k} .
$$

By symmetry this is also valid if $A, a$ and $B, b$ are interchanged. Thus we get that

$$
s=\sum_{j} a_{j} \sum_{i} A_{j}\left(b_{i}\right) B_{i}\left(a_{j}\right)-\sum_{i} b_{i} \sum_{j} A_{j}\left(b_{i}\right) B_{i}\left(a_{j}\right)=\sum_{j} a_{j}-\sum_{i} b_{i} .
$$


For the special case of the Hilbert segment (2), formula (13) gives Smith's [6] formula: $s=n(p+n)$ and for the matrix of (3) we get the formula of Collar [2]: $s=p n$.

3. Row and column sums. Let the row and column sums of $G$ be, respectively,

$$
\sum_{j} c_{i j}=\alpha_{i}, \quad \sum_{i} c_{i j}=\beta_{j}
$$

and define the diagonal matrices $D_{\alpha}, D_{\beta}$ by

$$
\begin{aligned}
& D_{\alpha}=\left[\alpha_{1}, \alpha_{2}, \cdots, \alpha_{n}\right] \\
& D_{\beta}=\left[\beta_{1}, \beta_{2}, \cdots, \beta_{n}\right] .
\end{aligned}
$$

Theorem 2. The matrix $H$ then satisfies the following relations:

$$
\begin{gathered}
\alpha_{i}=-\frac{A\left(b_{i}\right)}{B^{\prime}\left(b_{i}\right)}, \quad \beta_{j}=\frac{B\left(a_{j}\right)}{A^{\prime}\left(a_{j}\right)}, \\
H^{-1}=D_{\alpha} H^{T} D_{\beta} .
\end{gathered}
$$

Proof: Assuming (16) to be true we note that $c_{i j}$ can be written in the form

$$
c_{i j}=\frac{1}{b_{i}-a_{j}} \frac{A\left(b_{i}\right)}{B^{\prime}\left(b_{i}\right)} \frac{B\left(a_{j}\right)}{A^{\prime}\left(a_{j}\right)}=\frac{\alpha_{i} \beta_{j}}{a_{j}-b_{i}}
$$

which gives (17).

To prove the formula for $\alpha_{i}$ in (16) we need only show that

$$
\sum_{j} \frac{B\left(a_{j}\right)}{\left(a_{j}-b_{i}\right) A^{\prime}\left(a_{j}\right)}=1 .
$$

However for any function $f(x)$ we may write

$$
f\left[a_{1}, a_{2}, \cdots, a_{n}\right] \equiv \delta^{(n-1)} f(x)=\sum_{j} \frac{f\left(a_{j}\right)}{A^{\prime}\left(a_{j}\right)}
$$

where the left side of $(20)$ is the $(n-1)$ th divided difference of $f(x)$ with respect to the $a_{k}$ (see Milne-Thomson [7] p. 9). If we apply (20) to the polynomial $f(x)=$ $B(x) /\left(x-b_{i}\right)$ of degree $n-1$ we have that $\delta^{(n-1} f(x) \equiv$ constant. Since the coefficient of $x^{n-1}$ in $f(x)$ is $1,(19)$ is proved.

An alternate proof of (19) may be obtained, without divided differences, by noting that the left hand side of (19) is the sum of the residues of the function $B(x) / A(x)\left(x-b_{i}\right)$ at the $a_{j}$. (This, in fact, follows readily from (10).) However for a sufficiently large circle $C$ about the origin in the complex $x$-plane

$$
\frac{1}{2 \pi i} \int_{C} \frac{B(x)}{\left(x-b_{i}\right) A(x)} d x=1
$$

which proves (19).

The proof for the $\beta_{j}$ is obtained in the same manner from (19) with the roles of $A, a$ and $B, b$ interchanged, and the theorem is proved. (The formula (19), incidentally, represents an extension of one of the formulas of Collar [2] for the 
sum of a series. Extensions of other formulas given in [2] can likewise be obtained from (20) by specializing $f(x)$.)

\section{Remarks.} then

1.) We note that if $H$ is symmetric then $\alpha_{i}=\beta_{i}$ and if we set $D=D_{\alpha}=D_{\beta}$

$$
G=D H D
$$

2.) From (16) and (17) one immediately obtains the formula for the determinant of $H$ up to a sign. That is

$$
(\operatorname{det} H)^{2}=\prod_{i} \frac{1}{\alpha_{i} \beta_{i}}
$$

and for $H$ symmetric we get that

$$
\operatorname{det} H=(-1)^{[n(n-1)] / 2} \prod_{i} \frac{A^{\prime}\left(a_{i}\right)}{B\left(a_{i}\right)} .
$$

The signs may readily be determined, by induction, by using (6) and the formula for the $(n+1, n+1)$ element of $G_{n+1}$ :

$$
c_{n+1, n+1} \cdot \operatorname{det} H_{n+1}=\operatorname{det} H_{n}
$$

where

$$
H_{n}=H \quad \text { and } \quad H_{n+1}=\left\{\frac{1}{a_{i}-b_{j}}\right\} \text { with } 1 \leqq i, j \leqq n+1 .
$$

3.) Formula (8) may also be applied to the problem of obtaining a least-squares fit of a function $f(x)$ on, say, $(0,1)$ by a function of the form $\sum_{i} \gamma_{i} x^{a_{i}}$. (All variables are here assumed to be real.) The normal equations for this problem yield a matrix of the form (1) with $b_{i}=-a_{i}-1$, and the $\gamma_{i}$ may be obtained by applying $G$ to the moments of $f(x)$ with respect to the $x^{a_{i}}$.

For fitting a function of two variables the problem to determine $\gamma_{i j}$ such that

$$
\int_{0}^{1} \int_{0}^{1}\left[f(x, y)-\sum_{i, j} \gamma_{i j} x^{a_{i}} y^{a_{i}{ }^{\prime}}\right]^{2} d x d x=\min
$$

is solved by

$$
K=D H D F D^{\prime} H^{\prime} D^{\prime}
$$

where

$$
\begin{array}{r}
H=\left\{\frac{1}{a_{i}+a_{j}+1}\right\}, \quad H^{\prime}=\left\{\frac{1}{a_{i}^{\prime}+a_{j}^{\prime}+1}\right\}, \quad K=\left\{\gamma_{i j}\right\}, \\
F=\left\{\int_{0}^{1} \int_{0}^{1} f(x, y) x^{a_{i}} y^{a_{j^{\prime}}} d x d y\right\},
\end{array}
$$

and $D, D^{\prime}$ are the diagonal matrices corresponding to $H, H^{\prime}$, respectively, defined above. 
Although the elements $c_{i j}$ get quite large in the case of the Hilbert matrix, it may happen that for suitable choices of the $a_{i}$ this may not be the case.

An explicit solution for $\gamma_{i}$ can also be obtained from (7) for the equations

$$
\int_{0}^{1}\left(f(x)-\sum_{i} \gamma_{i} x^{a_{i}}\right) x^{a_{k^{\prime}}} d x=0, \quad k=1,2, \cdots, n
$$

given the moments of $f(x)$ with respect to the $x^{a_{i}{ }^{\prime}}$.

4.) If $H$ is real and symmetric and if $a_{i}>b_{i}, i=1,2, \cdots, n$ then it follows from (4) that all the principal minor determinants are positive and $H$ is positive definite. In this case $a_{i}>b_{j}$ for all $i$ and $j$; that is, all the elements of $H$ are positive, since if $a_{i}<a_{j}$, then $0<a_{i}-b_{i}<a_{j}-b_{i}$. Thus $B\left(a_{i}\right)>0$ and, since $A(x)$ has $n$ simple zeros at the $a_{i}, A^{\prime}\left(a_{i}\right)$ alternate in sign. From (8) it then follows that, if $a_{i}<a_{j}$ for $i<j$ then $(-1)^{i+j} c_{i j}>0$ so that $G$ has the same checkerboard distribution of signs as the inverse of the Hilbert segment. From (16) it follows that in this case the $\alpha_{i}$ also alternate in sign and that $(-1)^{j+n} \alpha_{j}>0$.

Let $\lambda$ and $\mu$ denote the smallest and largest eigenvalue of $H$ respectively, and let

$$
M_{i}=\min _{k, k \neq i}\left(\frac{a_{k}-b_{i}}{\left|a_{k}-a_{i}\right|}\right) .
$$

Then it follows readily that $\left|A_{i}\left(b_{i}\right)\right| \geqq M_{i}^{n-1}>1$ and that

$$
\lambda \leqq \min _{i} \frac{1}{c_{i i}} \leqq \min _{i}\left(\frac{M_{i}^{2-2 n}}{a_{i}-b_{i}}\right)
$$

If the $a_{i}$ increase with $i$ then

$$
\min _{i}\left(\sum_{j}(-1)^{i+j} c_{i j}\right)^{-1} \leqq \lambda .
$$

We then get for the $P$-condition [8] of $H$ :

$$
\frac{\mu}{\lambda} \geqq \max _{i}\left(\frac{1}{a_{i}-b_{i}}\right) \cdot \max _{i} c_{i i} \geqq \max _{i}\left[A_{i}\left(b_{i}\right)\right]^{2} \geqq \max _{i} M_{i}^{2 n-2}
$$

so that the $P$-condition of $H$ may get very large. This number has been estimated for the Hilbert segment (2) with $p=0$ by Todd [8].

New York University,

New York, New York

1. A. R. Collar, "On the reciprocation of certain matrices," Proc. Roy. Soc. Edin., v. 59, 1939 , p. 195-206.

2. A. R. Collar, "On the reciprocal of a segment of a generalized Hilbert matrix," Proc. Cambridge Philos. Soc., v. 47, 1951, p. 11-17.

3. E. H. Linfoot \& W. M. ShEPHERD, "On a set of linear equations, II," Quart. J. Math., Oxford, v. 10, 1939, p. 84-98.

4. I. R. SAVAGE \& E. LUKACS, "Tables of inverses of finite segments of the Hilbert matrix," Contributions to the Solution of Systems of Linear Equations, O. Taussky, Editor, National Bureau of Standards Applied Mathematics Series 39, 1954, p. 105-108. U. S. Govt. Printing Office.

5. G. P6LYa \& G. Szego, Aufgaben und Lehrsätze aus der Analysis, v. 2, Springer, Berlin, 1925, (Reprinted by Dover Publications, New York, 1945), p. 98.

6. R. B. SMITH, "Two theorems on inverses of finite segments of the generalized Hilbert matrix," MTAC, v. xiii, no. 65, January 1959.

7. L. M. Mrne-Thomson, The Calculus of Finite Differences, Macmillan, London, 1951.

8. J. TodD, "The condition of the finite segments of the Hilbert matrix," Contributions to the Solution of Systems of Linear Equations, O. Taussky, Editor, National Bureau of Standards Applied Mathematics Series 39, 1954, p. 109-116. U. S. Govt. Printing Office. 